\title{
Exposure Therapy with a Non-Verbal Child: A Case Report
}

\author{
By Timothy Zeiger* \\ Lidija Petrovic-Dovat \\ Pevitr S. Bansal
}

\begin{abstract}
Exposure Therapy (ET), a component of Cognitive-Behavioral Therapy (CBT), has long been used to treat psychiatric disorders including anxiety disorders, such as, Post-Traumatic Stress Disorder (PTSD), Obsessive-Compulsive Disorder, and, more recently, Selective Mutism. ET aims to reduce the levels of anxiety an individual experiences by gradually exposing them to said distressing stimulus. Although effective, this treatment type has not been applied to children suffering with a complex combination of genetic and psychiatric disorders, and intellectual disabilities. The following case study focuses on a 10-year-old girl with a history of medical illnesses including CHARGE syndrome, a rare genetic disorder. After an emergency surgical procedure, the child developed a myriad of psychiatric symptoms associated with PTSD and Selective Mutism after experiencing a stressful medical hospitalization. An exposure-based intervention was developed and implemented with frequency data pertaining to the symptoms were assessed prior, during, and post intervention. Outcome data were positive, demonstrating a decrease in the child's anxious behaviors after a single exposure-based session.
\end{abstract}

Keywords: CHARGE syndrome, Cognitive-behavioral, Exposure therapy PTSD.

\section{Introduction}

The application of cognitive behavior therapy (CBT) onto children with intellectual, developmental, and genetic disabilities is a relatively new and emerging field of study. Along with a plethora of medical issues, mental health also seems to be a concern for these individuals. Problems with anxiety have been reported as one of the most common forms of psychological distress for individuals with intellectual disabilities (Deb et al. 2001). Likewise, children with a certain autosomal dominant rare genetic condition are likely to be diagnosed with comorbid Obsessive-Compulsive Disorder (OCD), Autism, Attention/Deficit-Hyperactivity Disorder, and Tourette's syndrome (Bernstein and Denno 2005, Hartshorne et al. 2005, Waschtel et al. 2007).

This genetic disorder in question is CHARGE syndrome (Hall 1979), which is an acronym describing the disorder's abnormalities: Coloboma (keyhole slot in the iris, retina, and/or optic nerve); disorders with the Heart;

\footnotetext{
* Assistant Professor, Psychiatry, Penn State Milton S. Hershey Medical Center, USA.

${ }^{\dagger}$ Assistant Professor, Psychiatry, Penn State Milton S. Hershey Medical Center, USA.

* Penn State Milton S. Hershey Medical Center, Department of Psychiatry, USA.
} 
Atresia choanae (blocking of the breathing passages); Retarded growth and development; Genitourinary anomalies; and Ear and hearing anomalies. This genetic condition has an estimated incidence rate of 1 in 10,000 to 15,000 live births (Issekutz et al. 2005, Wachtel et al. 2007).

Many children with CHARGE syndrome display a set of challenging and problematic externalizing behaviors, including self-injury, aggression, and explosive/unstable behavior (Souriau et al. 2005, Thelin and Fussner 2005, Van Dijk and de Kort 2005). In addition to the aforementioned psychiatric (e.g., OCD, Autism), other disorders such as anxiety and depression (31\% and 24\% respectively) accompany CHARGE syndrome (Souriau et al. 2005). Repetitive behaviors are often characteristic of individuals with CHARGE but it should be noted that they are not a function of their sensory or communication impairment but of their CHARGE diagnosis (Bernstein and Denno 2005). There is limited literature on the treatment of behavioral issues within CHARGE syndrome. Bernstein and Denno (2005) have classified the repetitive behaviors into four categories: a) self-stimulatory; b) maladaptive; c) tics; and d) obsessive-compulsive. Treating these behaviors includes techniques include replacement of maladaptive behaviors with adaptive behaviors, rewards and consequences for certain behaviors, and implementing internal controls. Interventions utilizing CBT specifically, however, onto children with CHARGE syndrome is unavailable, suggesting that the treatment orientation has not been applied to the genetic disorder.

Exposure therapy (ET) is a form of CBT which places the patient in a position to confront fearful and frightening stimuli. This "exposure" to the distressing stimuli is repeated until anxiety levels are reduced (Rothbaum and Schwartz 2002, Neuner et al. 2008). ET has been found to have significant effects in reducing anxiety levels within various disorders including OCD, panic disorder, and phobias (Rothbaum and Schwartz 2002). Treatment gains from ET have been maintained for as long as two years post-treatment (Deblinger et al. 1999, Neuner et al. 2008). Anxious youth receiving treatment report the exposure, along with processing of the exposure, as an important process in reducing levels of anxiety (Tiwari et al. 2013).

In addition, ET had been utilized as a treatment approach onto children with Selective Mutism (SM). SM is a psychiatric disorder in which children show a consistent failure or inability to speak in certain situations while showing the ability to speak in other settings (Black and Udhe 1995). Although children with SM display negative characteristics such as aggression and oppositional behavior, their failure to speak does not stem from a refusal to do so (Cohan et al. 2006); instead SM has been found to be prevalent in children that have experienced psychological or physical trauma (Black and Udhe 1995). Sulkowski et al. (2014) utilized ET and other CBT components, such as psychoeducation and cognitive restructuring, onto a 12-year-old boy with selective mutism. Results demonstrated a positive effect of ET on the adolescent's symptoms. Once unable to speak with his family, with ET he was able to freely talk with his immediate and extended family, along with other individuals in the community (e.g., teachers, cashiers, and waiters). 
Given the above review, literature supports the use of CBT, ET, and other components, as successful treatments for children with various internalizing disorders including anxiety disorders, OCD, PTSD, and selective mutism. Many case studies have viewed ET's efficacy onto individuals with internalizing disorders; however, this case study may be the first to utilize and conduct ET onto a child with comorbid genetic and psychiatric disorders, including CHARGE syndrome, Post-Traumatic Stress Disorder (PTSD), and selective mutism.

\section{Case Report}

\section{Participant}

Jane Doe (pseudonym) is a 10-year-old Caucasian girl who lives with her mother and father. She is in fifth grade and receives special education in school. Her academic records were not available for review, but her parents believe that she is functioning on a level of a four-to-six year-old.

\section{Medical \& Psychiatric History}

Jane struggles with many features consistent with CHARGE syndrome, although she does not meet full diagnostic criteria. She was born with a heart defect but does not have atresia of choanal. Jane is blind in one eye but is fully capable of hearing in both ears. She has partial genital abnormalities. In addition to the CHARGE abnormalities, she was born with a hypoplastic right and a normal left kidney. Jane did not undergo any surgical procedures during her first three years of life, but does have a lengthy list of surgeries after age three. In April, 2014, at age 10, she developed an ileus and was admitted into pediatric surgery for 10 days, resulting in an ileostomy. Prior to this assessment, Jane did not have psychiatric assessment and had never been in behavioral therapy.

\section{Reason for Referral}

Behavioral concerns occurred when Jane was roughly nine-years-old during an October, 2012, hospitalization after receiving an emergency laparotomy. It is reported that the night after the surgery, a nurse's aide entered the room to perform a routine vital sign check-up on Jane. Jane awoke in a terrified manner as the nurse began her duties. There were no accounts of abusive behavior by the nurse's aide toward the child, per witness accounts. Since that night, Jane now experiences sleep disturbances, an overall irritated mood, an increased startle response, and psychomotor agitation, or feeling "jumpy". Furthermore, she is also withdrawn and not speaking to her family members. 
Jane's mother also noted multiple odd behaviors that she developed upon discharge. For instance, Jane began pulling hair and made inappropriate vocalizations with her mouth when in the presence of the nurse's aide and in the hospital setting. Neither behavior was present prior to the surgery. These behaviors have negatively affected the relationships she has with her friends and family; in addition, she now sleeps with a night-light. The aforementioned events and behaviors were described to the interviewer by Jane's mother. Jane communicates with the use of an electronic tablet-like device. This device allows Jane to push buttons that forms sentences for her, allowing appropriate and accurate expression of feeling. She was trained to use this device by her school.

\section{Provisional Diagnosis}

Child psychologists diagnosed Jane with the following, as per the Diagnostic and Statistical Manual for Mental Disorders Text-Revision, Fourth Edition (DSM-IV-TR, APA 2000):

- Axis I: Anxiety Disorder, NOS; Obsessive-Compulsive Disorder rule out Post-Traumatic Stress Disorder

- Axis II: Deferred

- Axis III: CHARGE syndrome; persistent left-sided superior Vena Cava; hypertension; post ileostomy

- Axis IV: Severe related to social environment; post recent surgery

- Axis V: 25

\section{Assessment}

The Child and Adolescent Outpatient unit within a large northeastern medical center was consulted by the pediatric surgery team post-hospital discharge, given that her anxious symptoms developed at discharge. The child and her family were initially seen by child psychiatrists to assess the nature of the concerns. Psychotropic medication was not thought to be clinically necessary, given the acute onset of the symptoms. The child was then referred to the clinical psychologist for further assessment.

During this initial intake, the child presented with symptoms consistent with PTSD. Conversely no traumatic event, as per diagnostic criteria, could be identified. The child's limited communication with her family members is congruent with Selective Mutism. The child was fearful that the nurse's aide would cause her, and other children in the hospital, physical discomfort. She experienced recurrent thoughts related to the hospital visit, and continued to exhibit a post-hospitalization fear response as evidenced by the behaviors and constant scanning of her environment. Jane was only able to concretely express her fear of nurse's aide, and ask of her whereabouts, through her communication device multiple times during the intake. 
Baseline frequencies of the anxious behaviors (e.g., hair-touching and vocalizations) were observed by a therapist during the intake psychotherapy session (Table 1). The hair-touching and vocal sounds occurred four to five times per minute, without mention of the hospitalization. When the hospitalization was mentioned, the hair-touching and vocal sounds nearly doubled, increasing to 5-10 times per minute. During a preferred activity (e.g., coloring) the behaviors desisted, occurring zero times per minute. An exposure session was planned with the mother and child during the intake psychotherapy session.

It is important to note that only behavioral observations of the anxious behaviors were gathered. Formal assessment measures of the symptoms were not administered given Jane's nonverbal presentation.

Table 1. Behavior Frequencies at Assessment

\begin{tabular}{|l|c|}
\hline Time Sample & $\begin{array}{c}\text { Behavior Occurrence } \\
\text { (\# of times/minute; \% range) }\end{array}$ \\
\hline Hospitalization NOT Mentioned & $4-5(6.7 \%-8.3 \%)$ \\
\hline Hospitalization Mentioned & $5-10(8.3 \%-16.7 \%)$ \\
\hline $\begin{array}{l}\text { During Preferred Activity } \\
\text { (E.g., Coloring) }\end{array}$ & $0(0 \%)$ \\
\hline
\end{tabular}

\section{Intervention}

The child psychologist discussed with the mother the importance of working with Jane in getting her to express herself through the communication device. After appropriate expression the mother would verbally reinforce the child's actions, and would reassure that she would be safe when meeting with the nurse. The psychologist believed that Jane would greatly benefit from "One-Session-Treatment". This ET concept combines behavioral, social, learning, and cognitive techniques into a single, massed session of graduated exposure. This concept allows the participant to gradually deal with their anxiety, at their own pace, within a controlled environment (Ost 1989).

The components of the "One-Session Treatment" included helping Jane to develop an initial state of relaxation while gradually exposing her to the feared stimulus. Throughout the exposure, the goal was to assess via behavioral observation her level of arousal and use behavioral strategies to help her regain a state of baseline relaxation while helping her overcome her fears.

The intervention was conducted in January, 2013. On the day of treatment the child psychologist, along with a service dog, greeted the child and family in the hospital lobby. The service dog helped ease Jane's level of anxiety as evidenced by her physiological and affective states (e.g., relaxed, smiling), and absence of maladaptive behaviors. The family, child, and psychologist returned to the floor in which Jane was originally hospitalized on. Certain objects revolving around the child's preferences were employed in order to maintain her reduced anxiety levels. For example, Jane's prefers the color blue, and therefore rode into the conference room on a blue wagon. 
Jane's communication device and other writing utensils and boards were made available to help with her self-expression throughout the exposure. Once all devices were available, the nurse's aide was requested into the conference room. Initial exposure to the nurse's aide engendered a high degree of fear within Jane, as evidenced by her clingy attachment to her mother in addition to her hair-touching and oral vocalizations. The nurse's aide remained on the opposite side of the room from Jane. Jane's mother reassured Jane of her safety and validated her bravery in the session which decreased Jane's anxiety levels. The CBT components of exposure and validation were repeated until Jane's anxiety levels remained consistently decreased, and began interacting with the aide.

\section{Results}

Pre- and post-treatment observations were made and calculated (Table 2). Initial exposure to the aide caused Jane to exhibit her negative behaviors approximately 5-10 times per minute. When her mother verbally reassured her, these behaviors decreased and occurred roughly zero to five times per minute. The child became more comfortable with the aide; her vocalization and hairtouching further diminished, occurring less than five times per minute.

Table 2. Behavior Frequencies at Pre-and Post-Treatment

\begin{tabular}{|l|c|}
\hline Time Sample & $\begin{array}{c}\text { Behavior Occurrence } \\
\text { ( of times/minute; \% range) }\end{array}$ \\
\hline $\begin{array}{l}\text { Pre-Treatment } \\
\text { (Exposure to Aide) }\end{array}$ & $5-10(8.3 \%-16.7 \%)$ \\
\hline $\begin{array}{l}\text { Post-Treatment } \\
\text { (Exposure to Aide) }\end{array}$ & $0-5(0 \%-8.3 \%)$ \\
\hline
\end{tabular}

Her physiological arousal subsided as evidenced by her smiling and the continued decrease of her distressing behaviors (e.g., hair-touching and vocal sounds). The child and nurse's aide engaged in many playful activities together. Pictures and videos of the child interacting with the nurse's aide were utilized as transitional objects. Replacement hand gestures (e.g., waving, drawing pictures) were developed to create a more positive association between the child, nurse's aide, and the hospital. By the end of the intervention, the child was accurately and consistently using the replacement hand gestures up to 5 times per minute.

\section{Conceptualization}

Jane's case can be viewed through classical conditioning. In classical conditioning, a conditioned stimulus (CS; stimulus causing neutral/no arousal) is paired with a conditioned response (UR; stimulus causing arousal). The repeating of this pairing leads to a conditioned response (CR; neutral stimulus 
now causes arousal) (Seligman 1971, Holland and Bouton 1999). In Jane's case, the CS was the nurse and the UR was the pain or fear from that occurred from the routine check-up. Given her stressful hospitalization, Jane began to fear the nurse's aide, associating her with the pain from the check-up. The child's fear became generalized to all settings outside of the hospital, that she began exhibiting the stress responses (hair-touching, producing vocal sounds) multiple times per hour post-hospitalization.

The exposure protocol was developed to help the child face and work through her fears related to the nurse's aide and hospitalization. Due to her cognitive and language delays the child had difficulty expressing her fears due to her cognitive and language delays. The child psychologist, along with Jane's family, was able to successfully establish concrete cognitive replacement strategies and replacement behaviors to aid in appropriate expression. Through multiple trials she began to independently utilize these replacement behaviors for the maladaptive behaviors.

\section{Discussion}

This case study is the first to conduct exposure therapy onto a child with a rare genetic disorder (e.g., CHARGE syndrome) and comorbid psychiatric issues (e.g., PTSD and selective mutism). This case focuses on a child that developed a surfeit of psychiatric symptoms after a frightening encounter with a nurse's aide. The issues include symptoms associated with anxiety, PTSD, and selective mutism, along with behaviors such as air-touching and making oral vocalizations when she was in the presence of the nurse's aide. A team of child psychologists implemented various CBT tactics including cognitive restructuring and behavioral management. The child responded rapidly and positively to the exposure-based treatment. She was able to freely speak with her family, and utilize replacement behaviors without verbal prompts from adults around her. She began sleeping better and reported an absence of nightmares than pre-treatment reports. These results were maintained during a six month post-treatment check-up.

Although the case study does highlight the success of treatment outcomes, there are a few limitations that must be addressed. A glaring limitation revolves around the lack of cognitive assessments. Jane's parents reported that she was functioning as a 4 -6-year-old, however the psychologists did not have access to her school records. Likewise, there was no testing of any sort to compute Jane's IQ, or assess for any intellectual disabilities. In addition, these interventions were not designed to improve the medical nature of the condition; any issues regarding the CHARGE syndrome were directed to medical professionals. Lastly, this case demonstrates CBT and ET's rapid and positive effects on a child with an assortment of health issues; however, further research must be conducted in order to build upon the external validity of these results. This single case study is not an appropriate sample size to generalize to the entire pediatric population suffering from comorbid medical and psychiatric 
issues. Further case studies should replicate these procedures on a similar population.

\section{Conclusion}

This case study highlights the positive effects of CBT and its components onto a child with a surfeit of medical and psychiatric issues. Many CBT techniques were utilized including cognitive restructuring, skill building, problem solving techniques, and exposure training. As aforementioned, gradually introducing an individual to an anxiety-producing stimulus actually reduces the individual's level of anxiety overtime (Lyneham and Rapee 2005). This was evident in Jane's case, as repeated exposure to the nurse's aide led to reduced levels of anxiety. Results from this case study support ET as an effective intervention for children with medical and psychiatric disorders. Further research and psychotherapeutic assessments should be conducted to view the trend of CBT's effects onto this specific population.

\section{Acknowledgments}

The authors would like to thank Neel Nene, M.D., Farhat Siddiqui, M.D., \& Diane Jasmine, D.O., for their contribution towards this case study.

\section{References}

American Psychiatric Association (APA) (2000). Diagnostic and statistical manual of mental disorders (4th ed., text rev.). Washington, DC: Author.

Bernstein V, Denno LS (2005) Repetitive behaviors in CHARGE syndrome: Differential diagnosis and treatment options. American Journal of Medical Genetics Part A 133(3): 232-239.

Black B, Uhde TW (1995) Psychiatric characteristics of children with selective mutism: a pilot study. Journal of the American Academy of Child \& Adolescent Psychiatry 34(7): 847-856.

Cohan SL, Chavira DA, Stein MB (2006) Practitioner review: Psychosocial interventions for children with selective mutism: A critical evaluation of the literature from 1990-2005. Journal of Child Psychology and Psychiatry 47(11): 1085-1097.

Deb S, Thomas M, Bright C (2001) Mental disorder in adults with intellectual disability. 1: Prevalence of functional psychiatric illness among a community-based population aged between 16 and 64 years. Journal of Intellectual Disability Research 45(6): 495-505.

Deblinger E, Steer RA, Lippmann J (1999) Two-year follow-up study of cognitive behavioral therapy for sexually abused children suffering post-traumatic stress symptoms. Child Abuse \& Neglect 23(12): 1371-1378.

Hall BD (1979) Choanal atresia and associated multiple anomalies. Journal of Pediatrics 95(3): 395-398. 
Hartshorne TS, Grialou TL, Parker KR (2005) Autistic-like behavior in CHARGE syndrome. American Journal of Medical Genetics Part A 133(3): 257-261.

Holland PC, Bouton ME (1999) Hippocampus and context in classical conditioning. Current opinion in neurobiology 9(2): 195-202.

Issekutz KA, Graham JM, Prasad C, Smith IM, Blake KD (2005) An epidemiological analysis of CHARGE syndrome: preliminary results from a Canadian study. American Journal of Medical Genetics Part A 133(3): 309-317.

Lyneham HJ, Rapee RM (2005) Evaluation and treatment of anxiety disorders in the general pediatric population: a clinician's guide. Child and Adolescent Psychiatric Clinics of North America 14(4): 845-861.

Neuner F, Catani C, Ruf M, Schauer E, Schauer M, Elbert T (2008) Narrative exposure therapy for the treatment of traumatized children and adolescents (KidNET): from neurocognitive theory to field intervention. Child and adolescent psychiatric clinics of North America 17(3): 641-664.

Ost LG (1989) One -session treatment for specific phobias. Behavior Research and Therapy 27: 1-7.

Rothbaum BO, Schwartz AC (2002) Exposure therapy for posttraumatic stress disorder. American journal of psychotherapy 56(1): 59.

Seligman ME (1971) Phobias and preparedness. Behavior therapy 2(3): 307-320.

Souriau J, Gimenes M, Blouin C, Benbrik I, Benbrik E, Churakowskyi A, Churakowskyi B (2005) CHARGE syndrome: developmental and behavioral data. American Journal of Medical Genetics Part A 133(3): 278-281.

Sulkowski M, Pence S, Carlson J, Storch E (2014) Treating Selective Mutism with Exposure Therapy: A Case Study. Annals of Psychotherapy.

Thelin JW, Fussner JC (2005) Factors related to the development of communication in CHARGE syndrome. American Journal of Medical Genetics 133A: 282-290.

Tiwari S, Kendall PC, Hoff AL, Harrison JP, Fizur P (2013) Characteristics of exposure sessions as predictors of treatment response in anxious youth. Journal of Clinical Child \& Adolescent Psychology 42(1): 34-43.

Van Dijk JPM, de Kort A (2005) Reducing challenging behaviors and fostering efficient learning of children with CHARGE syndrome. American Journal of Medical Genetics 133A: 273-277.

Wachtel LE, Hartshorne TS, Dailor AN (2007) Psychiatric diagnoses and psychotropic medications in CHARGE syndrome: A pediatric survey. Journal of Developmental and Physical Disabilities 19(5): 471-483. 
